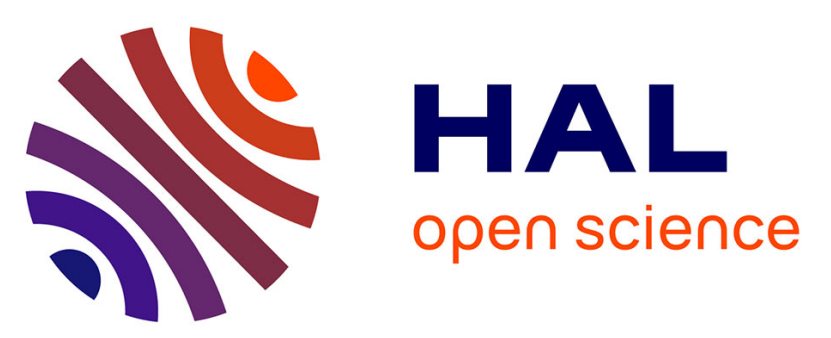

\title{
Time-dependent cognitive and somatic symptoms of depression as predictors of new cardiac-related events in at-risk patients: the UPBEAT-UK cohort
}

Joanna Norton, M Pastore, Marie-Laure Ancelin, M Hotopf, A Tylee, A.

Mann, J Palacios

\section{To cite this version:}

Joanna Norton, M Pastore, Marie-Laure Ancelin, M Hotopf, A Tylee, et al.. Time-dependent cognitive and somatic symptoms of depression as predictors of new cardiac-related events in at-risk patients: the UPBEAT-UK cohort. Psychological Medicine, In press, 10.1017/S0033291719004082 . hal-03139263

\section{HAL Id: hal-03139263 https://hal.science/hal-03139263}

Submitted on 25 Feb 2021

HAL is a multi-disciplinary open access archive for the deposit and dissemination of scientific research documents, whether they are published or not. The documents may come from teaching and research institutions in France or abroad, or from public or private research centers.
L'archive ouverte pluridisciplinaire HAL, est destinée au dépôt et à la diffusion de documents scientifiques de niveau recherche, publiés ou non, émanant des établissements d'enseignement et de recherche français ou étrangers, des laboratoires publics ou privés. 
Time-dependent cognitive and somatic symptoms of depression as predictors of new cardiac-related events in at-risk patients: the UPBEAT-UK cohort

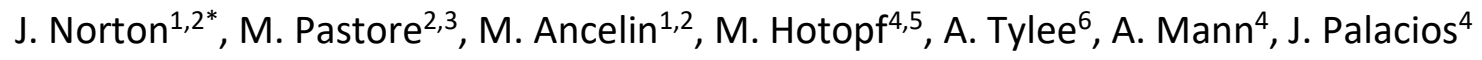

${ }^{1}$ Inserm U1061, Montpellier, France.

2 University of Montpellier, Montpellier, France.

${ }^{3}$ StatABIO, CNRS, INSERM, Montpellier, France.

${ }^{4}$ Department of Psychological Medicine, Institute of Psychiatry, Psychology and Neuroscience, King's College London, London, UK.

5 South London and Maudsley NHS Foundation Trust, London, UK.

${ }^{6}$ Department of Health Services and Population Science, Institute of Psychiatry, Psychology and Neuroscience, King's College London, London UK.

*Corresponding author

Inserm U1061, Neuropsychiatry: epidemiological and clinical research

Hôpital La Colombière Pav. 42

39 av. Charles Flahault

34093 Montpellier Cedex 5, France

Phone: +33499614570 ; Fax: +33499614579

joanna.norton@inserm.fr

4159 Words 
3 Tables,

1 Supplementary Table

2 Supplementary Figures 


\section{Abstract}

Background: evidence suggests that somatic rather than cognitive depressive symptoms are risk factors for recurrent cardiac events in at-risk patients. However, this has never been explored using a time-dependent approach in a narrow time-frame, allowing for an cardiac event-free time-window.

Methods: the analysis was performed on 595 participants $(70.6 \%$ male, median age 72 (2798)) drawn from the UPBEAT-UK heart disease patient cohort with 6-monthly follow-ups over 3 years. Depressive symptomatology was measured using the Patient Health Questionnaire-9 (PHQ-9) (four somatic, five cognitive items). New cardiac events (NCE) including cardiac-related mortality were identified by expert examination of patient records. Analyses were performed using Cox Proportional Hazard models with delayed entry, with time-dependent depressive dimensions and covariates measured 12-to-18 months (median: 14.1, IQR: 3.5) prior to the event, with a 12-month cardiac event-free gap.

Results: there were 95 NCE during the follow-up (median time-to-event from baseline: 22.3 months (IQR: 13.4)). Both the somatic (HR: 1.12, 95\% Cl.: 1.05-1.20, p=0.001) and cognitive dimensions (HR: 1.11, 95\% Cl.: 1.03-1.18, $\mathrm{p}=0.004$ ) were time-dependent risk factors for a NCE in the multi-adjusted models. Specific symptoms (poor appetite/overeating for the somatic dimension, hopelessness and feeling like a failure for the cognitive dimension) were also significantly associated.

Conclusion: This is the first study of the association between depressive symptom dimensions and NCEs in at-risk patients using a time-to-event standardized approach. Both dimensions considered apart were independent predictors of a NCE, along with specific items, suggesting regular assessments and tailored interventions targeting specific depressive symptoms may help to prevent new cardiac events in at-risk populations. 


\section{Introduction}

There is extensive research on the bi-directional relationship between depression and heart disease and the possible mechanisms involved (Ormel and de Jonge, 2011), and it is now widely acknowledged that depression is a risk factor for recurrent cardiac events and cardiac-related deaths in patients with heart disease (Freedland and Carney, 2013, Meijer et al., 2011).

Regarding specific depressive symptoms, a recent meta-analysis suggested that the somatic rather than the cognitive symptom dimension of depression was predictive of recurrent cardiovascular events in at-risk patients (de Miranda Azevedo et al., 2014). For instance, in a study of 2347 acute myocardial infarction (MI) patients, the Patient Health Questionnaire-9 (PHQ-9) somatic symptom dimension only was independently associated with one-year rehospitalisation and to a lesser extent 4-year mortality (Smolderen et al., 2009). In keeping with this, in a cohort of 1019 stable coronary heart disease (CHD) patients, a 14\% greater risk of cardiovascular events over 6 years was reported for the PHQ-9 somatic dimension and, more specifically, for fatigue and appetite problems (Hoen et al., 2010b). Several other studies also found somatic symptoms only to be associated with cardiac health status, cardiac prognosis and mortality in MI patients (de Jonge et al., 2006, Martens et al., 2010, Roest et al., 2011). In contrast, some studies identified as potential risk factors for a recurrent event either both dimensions (Denollet et al., 2013, Hoen et al., 2010a) or the cognitive dimension only when depressive symptoms were evaluated postoperatively in coronary bypass patients (Connerney et al., 2010, Tully et al., 2011). However, the diversity of assessment scales and depressive symptom classifications makes study comparison difficult (Carney and Freedland, 2012b). 
There are various explanations for the widespread evidence in favour of somatic symptoms as risk factors for cardiac events. Firstly, somatic symptoms tend to be more common in depressed patients with heart disease compared to other depressed patients (Martens et al., 2006). They are more likely to overlap with cardiac disease, not only because some symptoms such as fatigue are shared by both conditions, but also because depressed at-risk patients may focus more on their somatic symptoms which they can attribute to their underlying heart condition (Carney and Freedland, 2012a). Secondly, somatic depressive symptoms are likely to be overestimated by self-report questionnaires on which findings almost exclusively rely. This is because no weighing of symptoms is performed as with structured diagnostic interviews. In the only study assessing depression using a diagnostic interview, both somatic and cognitive symptoms were predictive of cardiovascular prognosis in the multi-adjusted analysis (Hoen et al., 2010a). Thirdly, studies performed so far have not addressed the time-frame of the exposure-event matrix. In most studies depressive symptomatology was assessed at a fixed time-point with considerable variability in duration of time between assessment and cardiac onset (Connerney et al., 2010, Hoen et al., 2010b). The absence of a cardiac event-free time-window between depression assessment and outcome could lead to a greater reporting of somatic symptoms, as markers of cardiac disease severity (Meijer et al., 2013).

Depressive symptoms have been shown to fluctuate in the elderly, especially over the longterm, and persistent high or increasing symptom trajectories have been linked to adverse health outcomes, such as dementia (Kaup et al., 2016) and mortality (Andreescu et al., 2008). However, irrespective of symptom change, symptom levels per se can exhibit timevarying effects; this has been shown for example for all-cause and non-cardiovascular disease-specific mortality over a 7-year follow-up (Moise et al., 2018). A time-dependent 
approach allows the assessment of risk within a defined time-window, standardising length of time between exposure assessment and the outcome. The potential time-varying effect of the exposure is thereby taken into account. Studies analysing depression as a timedependent risk factor are scarce (Li et al., 2018, Pequignot et al., 2016). Over a 12-year follow-up, Li et al. found that contrary to baseline depressive symptoms, time-dependent symptoms increased the risk of all-cause and cardiovascular mortality (Li et al., 2018). However, no studies so far have examined the different symptom dimensions as recurrent cardiac event risk factors using this approach.

The aim of the current study was to examine PHQ-9 somatic and cognitive symptom dimensions, measured 12 to 18 months prior to the event, as short-term predictors of cardiac-related events and mortality, drawing on data from the UPBEAT-UK cohort of heart disease patients followed up at 6-monthly intervals over 3 years. Individual symptoms and symptom change were also investigated.

\section{Methods}

\section{Study design and sample}

This study draws on data from the UPBEAT-UK cohort. Participants were identified and recruited from Coronary Heart Disease (CHD) Registers kept by the 16 participating general practices in South East London (Tylee et al., 2011). All register patients ( $n=2938)$ were invited to participate by their local GP; 917 agreed to be contacted, of which 803 (87.6\%) participated. Each participant had an initial baseline interview and was followed up for three years, undergoing assessments every six months. Recruitment and assessments ran from 2008 to 2011 . Written informed consent was obtained from all participants before the initial 
assessment. Ethics approval was granted through the Bexley and Greenwich Research Ethics Committee (REC reference number: 07/H0809/38).

The present analysis was performed on 595 patients. From the initial 803 participants, we excluded 83 with an NCE recorded at cohort inclusion or at the 6 or 12 month follow-ups; a further 71 with outcome data missing at all four follow-ups (18, 24, 30 and 36 months), a further 25 with a delay between CHD register entry (corresponding approximately to the first major cardiac event) and recruitment into the cohort less or equal to 6 months, and a further 29 participants with missing data for any of the main covariates.

\section{Measures}

Socio-demographic and lifestyle characteristics, including relationship status, smoking, alcohol consumption and body mass index (BMI), were collected at baseline only. The Clinical Interview Schedule Revised (CIS-R) (Lewis et al., 1992) was administered to establish International Classification of Diseases-10 (ICD-10) diagnoses of depression at baseline. Each participant was attributed an Index of Multiple Deprivation (IMD) score according to their postcode (National Statistics, 30 September 2015).

At baseline and each follow-up, severity of chest pain was measured using the Rose Angina Questionnaire (Rose, 1962) and was classified as follows: no angina; chest pain when walking uphill only; chest pain when walking on the level and uphill. Anxiety scores were derived from the anxiety module of the Hospital Anxiety and Depression Scale (HADS-A) (Zigmond and Snaith, 1983). Time-dependent quality of life scores were obtained using the EuroQol EQ-5D scale, excluding the pain/discomfort and depression/anxiety subscales (Rabin and de Charro, 2001), and social problems from the Social Problems Questionnaire (SPQ) (Corney and Clare, 1985). 
Outcome variable: new cardiac event (NCE).

This was a composite of any cardiac event encompassing: any visit to the rapid access chest pain clinic, A\&E (Accident and Emergencies) or emergency hospital admittance, where cardiac-related chest pain was the diagnosis; bypass graft or angioplasty; MI and any cardiovascular cause of death. Events were coded and dated by a medical doctor on the team after examination of GP notes. Mortality data were gathered from case notes and other publically available sources. Cause and date of death were obtained from the practice manager at the different GP surgeries. For the purpose of the analysis, we refer to the first cardiac event noted at the $18,24,30$ and 36 month follow-ups, which occurred any time during the six months preceding the follow-up.

Exposure variable: somatic and cognitive depression dimensions.

These were derived from the PHQ-9, a screener measuring nine depressive symptoms over the past two weeks, each scored from 0 (not at all) to 3 (nearly every day) (Kroenke et al., 2001). Four questions refer to the somatic dimension (trouble with sleep, feeling tired, poor appetite/overeating, slowness/restlessness) with scores ranging from 0 to 12 . Five questions refer to the cognitive dimension (little interest in doing things, feeling depressed/hopeless, like a failure, trouble concentrating, thoughts of death), ranging from 0 to 15 . Scores were obtained by summing the individual items for each dimension.

\section{Statistical analysis}

All statistical tests were performed using Cox proportional hazard models with delayed entry corresponding to age at baseline, with age as the basic time scale and birth as the time 
origin. This method enables a better adjustment for age and is preferred, especially for elderly samples, over the standard model with time since inclusion as the time scale (Lamarca et al., 1998). The assumptions of proportional hazards over time for baseline variables and the linearity of all continuous variables were verified. Results are expressed as hazard ratios (HR) with 95\% confidence intervals $(\mathrm{Cl})$. For the depression dimensions, HRs represent the increased risk per one-point increase in score on an ordinal scale of 0-12 and $0-15$, for the somatic and cognitive dimensions respectively. For each individual symptom, this corresponds to a one-point increase on the 0-3 scale.

Somatic and cognitive dimensions, along with individual symptom items, were analysed separately both as baseline and time-dependent variables. We excluded participants with a NCE at the baseline, 6- and 12-month follow-ups. This ensured a gap of at least 12-months between baseline depression assessment and date of outcome. In the time-dependent analysis, depressive symptom scores and available covariates were taken at the $\mathrm{N}-3$ followup, allowing for this 12-month NCE-free gap. The median time between time-dependent measures and event was 14.1 months (Inter-quartile range (IQR): 3.5). Covariates measured at follow-up were: chronic diseases, chest pain, social problems, quality of life and anxiety. All other variables were available at baseline only. For the multivariate models, we selected all baseline or time-dependent covariates associated with NCE with $p$-values $\leq 0.25$ or considered as well-established risk factors in the literature (see Supplementary Table 1). Interactions with sex and chest pain were tested and not significant. Bonferroni correction was used to account for multiple comparisons when considering the individual symptoms on each depression dimension $(p<0.0125$ and $p<0.01$ for the somatic and cognitive dimensions, respectively). 
In addition, we studied time-dependent change in somatic and cognitive dimension scores since the previous follow-up using the same risk factor to event-time framework. For each subject, we calculated the relative change in score (score at time ' $\mathrm{t}$ ' minus score at time ' $\mathrm{t}-\mathrm{1}$ ' divided by score at ' $t-1$ ') on each dimension. We then compared the two extreme categories of the quartiles of the score distribution, with a greater than $25 \%$ decrease (i) or increase in score (ii), to the middle category representing the interquartile range (iii). Significance was set at $p<0.05$. Statistical analyses were performed using SAS Enterprise Guide Version 7.15 (SAS Institute, Inc. Cary, North Carolina).

\section{Results}

\section{Sample description}

The characteristics of the cohort have been described in detail elsewhere (Walters et al., 2014). We compared the study sample of 595 participants to the 208 participants excluded from the analysis. There were no differences for the main socio-demographic, lifestyle and health variables except for quality of life $(p=0.01)$, anxiety $(p=0.006)$ and the cognitive depression dimension $(p=0.0006)$, with higher severity scores for those excluded from the analysis.

The study sample was mainly male (70.6\%), with a median age of 72 years, ranging from 27 to 98 . Chest pain (Grade 1 or 2 angina) was reported at baseline by $24.1 \%$ of the sample. Depression was diagnosed in $5.9 \%$ of participants and $16.8 \%$ had a PHQ-9 score $>10$. The median (min-max) scores on the somatic and cognitive dimensions were $2(0-12)$ and 0 (012), respectively (Table 1$)$.

Of the sample, $42.4 \%$ entered the $\mathrm{CHD}$ register with a diagnosis of $\mathrm{MI}, 48.2 \%$ with ischemic heart disease, $6.9 \%$ with angina, and $2.5 \%$ with one of the following indications: heart 
failure, chest pain not otherwise specified, arrhythmias or other vascular problems. Median time between CHD register entry and baseline was 8.3 years, ranging from 0.6 (by design, as participants with a delay between CHD register entry and cohort entry less or equal to 6 months were excluded) to 42.8 years. Over the four follow-ups where outcomes were considered in the analysis, there were $95 \mathrm{NCE}, 31$ at the 18-month, 23 at the 24-month, 12 at the 30-month and 29 at the 36-month follow-up. Median time from baseline to event was 22.3 months (IQR: 13.4, min-max: 12.1-36.2). The most frequent event was a visit to the rapid access chest pain clinic, A\&E or emergency hospital admittance (47.4\%), followed by cardio-cerebrovascular cause of death (26.3\%), bypass graft or angioplasty (24.2\%), and MI (2.1\%).

Somatic and cognitive symptom dimensions as NCE risk factors

In the multivariate models, both the somatic and cognitive dimensions were significantly associated with a NCE whether measured as time-dependent variables or at baseline (Table 2). All analyses became non-significant when further adjusting for quality of life and anxiety, except for the somatic dimension at baseline $(p=0.04)$ (not shown).

Individual symptom items as NCE risk factors

Combining the baseline and time-dependent approaches, all individual somatic symptoms were significantly associated with the onset of a NCE in the multivariate models (Table 3). However, when applying Bonferroni correction, only poor appetite/overeating remained significant. Of the cognitive items, feeling down at baseline and feeling like a failure for the time-dependent approach were significant multiple-comparison corrected predictors. 
Time-dependent change in dimension scores as NCE risk factors

For both dimensions, variability in scores over follow-up were low and not significant (Fvalue $=0.32, p=0.93$ for the somatic dimension; $F$-value $=1.34, p=0.24$ for the cognitive dimension). Approximately one third of participants for the somatic dimension and half for the cognitive dimension presented no change in score between two-successive follow-ups (Figures 1 and 2). Time-dependent change in somatic and cognitive scores were not associated with the risk of a NCE.

\section{Discussion}

To the best of our knowledge, this is the first study to examine somatic and cognitive dimensions of depression as time-dependent risk factors for recurrent cardiac events. Conversely to findings from elsewhere, our findings suggest that both dimensions could be important short-term predictors of recurrent cardiac events when measured 12 to 18 months prior to the event, in particular somatic symptom appetite/overeating and cognitive symptoms feeling low and feeling like a failure.

\section{Cognitive and somatic symptom dimensions}

Our findings from the baseline and time-dependent analysis suggest both the somatic and cognitive depression dimensions to be predictors of a NCE, whereas most other studies find an effect for the somatic dimension only (de Miranda Azevedo et al., 2014). We adjusted, as elsewhere (Hoen et al., 2010b, Linke et al., 2009, Martens et al., 2010) for history of heart disease taking diagnosis at entry into the CHD register as a proxy, along with the main chronic diseases, smoking and chest pain. However, the associations became largely nonsignificant when further adjusting for quality of life and anxiety. Despite removing the 
pain/discomfort and anxiety/depression scales from the EQ-5D, they could be over-adjusted given the strong associations between chronic diseases and quality of life and the overlap in symptoms between subthreshold depression and anxiety (Braam et al., 2014). In fact, to our knowledge, no other study adjusted for these covariates in their multivariate models.

The findings for the time-dependent analysis are very similar to those using the baseline data. This is not actually surprising given that both analyses examined depressive symptoms as short-term risk factors for a NCE. The main difference between the two approaches is the variability in time-to-event (median (IQR): 22.3 (13.4) in the baseline analysis, and 14.1 (3.5) for the time-dependent analysis). Furthermore, we allowed for an event-free time-window between exposure and event in both approaches, in order to avoid the concomitant onset of the two, with depressive symptoms acting more like a cardiac disease marker than a shortterm risk factor.

The association between time-dependent depression and cardiac prognosis has seldom been investigated (Li et al., 2018, Pequignot et al., 2016). In a Chinese cohort of 1999 subjects aged 55 years and above, the time-dependent depressive symptom score increased the risk of all-cause and cardiovascular mortality over a 12-year follow-up, whereas no association was found with symptom score at baseline (Li et al., 2018). The difference could be explained by the longer follow-up period than in our study, with a greater difference in intersubject variability in time-to-event. In the Three-City Study including 9294 persons aged 65 years and above followed up for over 10 years, time-dependent number of visits with a high level of depressive symptoms was associated with cardiovascular disease mortality risk, which increased 1.28-fold for each additional visit (Pequignot et al., 2016). However, neither 
of these studies using the Centre for Epidemiologic Studies-Depression Scale considered somatic and cognitive symptom dimensions separately.

Many behavioural and biological mechanisms have been suggested to explain the link between depressive symptomatology and recurrent cardiac events. Inflammation is thought to play a key role, in particular in somatic and late-onset depressive symptoms (Meijer et al., 2013). Ormel and de Jonge suggested a differential effect on acute coronary syndromes in CHD patients, depending on the type of symptoms (Ormel and de Jonge, 2011). Their integrative model rests on three core hypotheses. The first is that depression in CHD patients consists of a mix of two types of depression, cognitive/affective and somatic depression, each having a characteristic symptom expression and aetiology. The second is that the effects on CHD depend on the type and duration of depression. The third hypothesis is that the most plausible pathways mediating these effects are behavioural. They argue that for both cognitive-affective and somatic depressive dimensions, persistent (chronic or recurrent) symptoms would be expected to be associated with recurrent cardiac events, but not brief symptom episodes (remission within 6 months) that are likely to be confounded by the severity of underlying cardiac symptoms and associated physiological abnormalities. Moreover, they suggest a different pathway for each symptom dimension, with behavioural factors alone mediating the cognitive pathway, whereas both behavioural and other factors such as inflammation would mediate the somatic symptom pathway.

Greater fluctuations over time of behavioural factors (e.g. lifestyle, medication adherence) compared to biological factors could explain why studies considering depressive symptoms at a fixed time-point find no association for the cognitive dimension. The latter present considerable variability, both between and within studies, in duration of time between baseline depression assessment and outcome. In the present study, median time to event 
was relatively short (22.3 months (IQR: 13.7)). Other studies report average times to event ranging from 2.5 years ( $\mathrm{sd}=0.9$ ) (de Jonge et al., 2006) to 6.1 years ( $\mathrm{sd}=2.0$ ) (Hoen et al., 2010b).

Of the few studies reporting an association for the cognitive dimension measured at a fixed time-point, Hoen et al. reported that both the somatic and cognitive dimensions were associated with cardiovascular prognosis in a study of $468 \mathrm{MI}$ patients with a relatively short mean follow-up of 2.5 years ( $s d=0.8$ ), (Hoen et al., 2010a). The authors attributed this to a more thorough assessment of symptoms thanks to the use of a structured diagnostic interview. This suggests that an overestimation of somatic symptoms with self-report instruments coupled with longer and varying times-to-event could account for previous negative findings for the cognitive dimension. Another study showed a link between the cognitive-affective dimension of the BDI administered at baseline and recurrent $\mathrm{MI}$ and mortality in 1823 patients with a mean follow-up of 2.1 years $(s d=1.1)$; this was found for the under 70 age-group only in a sample of patients with post-MI depression (Denollet et al., 2013). This highlights the complexity of the relationship between NCE and depressive symptoms that could be modulated by both an age and depression level threshold effect.

\section{Time-dependent symptom change over time}

We also examined for each dimension, the association between time-dependent symptom change and the risk of a NCE. Neither of the associations were significant. Of the few studies investigating change over time (Roest et al., 2013, Scafato et al., 2012), only Roest et al. with the ENRICHD study examined separate symptom dimensions. They reported that over 6 months improvement in somatic symptoms but not cognitive symptoms was associated with a reduced risk of recurrent $\mathrm{MI}$ and mortality; however, change was not considered as a time- 
dependent variable allowing for considerable variability in time-to-event over the 2.4 year (standard deviation $(s d)=2.9$ ) follow-up period (Roest et al., 2013).

We chose to express change in terms of the relative change in score but this may have biased our findings as nearly half of subjects were in the 'no change' category, in particular for the cognitive dimension due to a clustering of scores at 0 for approximately $50 \%$ of participants at each follow-up. Furthermore, the 'no change' category included the middle quartiles with both persistently high and low scores. This low level of change has previously been reported for this cohort (Palacios et al., 2016). A more sophisticated approach to change taking into account trajectories over the entire follow-up (Palacios et al., 2018), was not possible given the time-dependent approach used in the analysis.

\section{Individual depressive symptoms}

To our knowledge, this is the first time individual depressive symptom items are examined as time-dependent variables. Of the somatic symptoms, poor appetite/overeating was the most robust predictor of a NCE. Hoen et al. in a study of 1019 patients with stable CHD over a relatively long follow-up period (mean 6.1 years $(S D=2.0)$ ), found that only two of PHQ-9 symptoms - namely somatic items appetite problems and fatigue - measured as continuous variables at a fixed time-point were associated with recurrent cardiovascular events (Hoen et al., 2010b). When using a structured diagnostic interview, the same authors reported that all baseline somatic symptoms except for sleeping difficulties were predictors of cardiovascular prognosis, along with the cognitive symptoms lack of interest and suicidal ideation (Hoen et al., 2010a). Denollet et al. (2013) examined individual cognitive symptoms and found that only hopelessness remained significantly associated with recurrent $\mathrm{MI}$ and mortality 
(Denollet et al., 2013). This is in keeping with our findings regarding cognitive items feeling down and feeling like a failure.

\section{Limitations}

There are several limitations to our study. Firstly, we used a self-report instrument to measure depressive symptoms which may have led to an overestimation of somatic symptoms (Hoen et al., 2010a). On the other hand, the PHQ-9 has been suggested as a useful screening tool in cardiac patients, as it covers a wide range of symptoms with a clear and validated distinction between the somatic and cognitive dimensions (Carney and Freedland, 2012a, de Jonge et al., 2007). It has also been shown to be have a superior diagnostic accuracy to the HADS-D in CHD patients (Haddad et al., 2013). Secondly, although we were able to adjust for a wide range of variables, we did not have data on medication use and could not adjust for antidepressant or cardiac-related medication and treatment compliance. A further limitation is the heterogeneity of cardiac events both at entry into the CHD register and as outcome variable (Freedland and Carney, 2013). Indeed NCE included events ranging from rapid access chest pain clinic for cardiac-related chest pain to cardiocerebrovascular cause of death. The exclusion of events at the first three time-points and the limited number of events per category of NCE did not allow us to investigate each type of event separately. Finally, we were not able to show any effect of change in scores between follow-ups, mainly because symptom scores fluctuated very little over the followup. This in turn can be explained by the clustering of scores at the lower end of the distribution due to the selection of a healthy sub-sample, as well as the short duration of follow-up (36 months). 


\section{Strengths}

The main strength of our study is the design regarding the delay between depression assessment and the NCE. The time-dependent approach allowed us to standardise for timeto-event, which is crucial when examining symptoms that are known to fluctuate considerably over time. Furthermore, in order to distinguish 'depression' as a short-term risk factor for a NCE from 'depression as a non causal variable risk marker' (Meijer et al., 2013) we chose to leave an event-free period of 12-months between symptom assessment and the cardiac onset period. This reduced the likelihood of over-reporting somatic symptoms as a marker of cardiac disease.

We also removed patients who entered the register in the six months preceding baseline, in an attempt to measure depressive symptomatology as a risk factor for a NCE rather than the opposite, although we cannot fully rule out that baseline symptoms could be the consequence of past cardiac events or worsening of symptoms. Finally, our study was based on a relatively large sample of patients from CHD registers with regular 6-monthly follow-ups over 3 years, with full datasets. NCE cases were certified by an expert clinical review of all patient records with exact dates for events.

\section{Conclusion}

We showed that both the somatic and cognitive depressive symptom dimensions measured 12 to 18 months earlier were significantly and positively associated with a greater risk of a NCE. Thus, cognitive symptoms should not be disregarded as short-term risk factors for recurrent cardiac events as suggested by most previous research. More regular assessment of depressive symptoms, with a special attention given to specific symptoms such as eating 
problems and feeling hopeless or like a failure, could help predict and prevent future cardiac events.

\section{Acknowledgements}

The authors are grateful to the Primary Care Research Network-greater London for their support and assistance in recruiting practices to the UPBEAT-UK study and to the 16 practices who provided assistance. They are also grateful for the work of the UPBEAT research team for setting up the study and collecting the data.

\section{Financial support}

This article represents independent research commissioned by the National Institute for Health Research (NIHR) under it Programme Grants for Applied Research scheme (RP-PG0606-1048). It also represents independent research part-funded by the National Institute for Health Research (NIHR) Biomedical Research Centre at South London and Maudsley NHS Foundation Trust and King's College London. The views expressed are those of the authors and not necessarily those of the NHS, the NIHR or the Department of Health.

\section{Conflict of interest}

None

\section{References}


Andreescu, C., Chang, C. C., Mulsant, B. H. \& Ganguli, M. (2008). Twelve-year depressive symptom trajectories and their predictors in a community sample of older adults. Int Psychogeriatr 20, 221-36.

Braam, A. W., Copeland, J. R., Delespaul, P. A., Beekman, A. T., Como, A., Dewey, M., Fichter, M., Holwerda, T. J., Lawlor, B. A., Lobo, A., Magnusson, H., Prince, M. J., Reischies, F., Wilson, K. C. \& Skoog, I. (2014). Depression, subthreshold depression and comorbid anxiety symptoms in older Europeans: results from the EURODEP concerted action. J Affect Disord 155, 266-72.

Carney, R. M. \& Freedland, K. E. (2012a). Are somatic symptoms of depression better predictors of cardiac events than cognitive symptoms in coronary heart disease? Psychosom Med 74, 33-8.

Carney, R. M. \& Freedland, K. E. (2012b). Is there a high-risk subtype of depression in patients with coronary heart disease? Curr Psychiatry Rep 14, 1-7.

Connerney, I., Sloan, R. P., Shapiro, P. A., Bagiella, E. \& Seckman, C. (2010). Depression is associated with increased mortality 10 years after coronary artery bypass surgery. Psychosom Med 72, 874-81.

Corney, R. H. \& Clare, A. W. (1985). The construction, development and testing of a self-report questionnaire to identify social problems. Psychol Med 15, 637-49.

de Jonge, P., Mangano, D. \& Whooley, M. A. (2007). Differential association of cognitive and somatic depressive symptoms with heart rate variability in patients with stable coronary heart disease: findings from the Heart and Soul Study. Psychosom Med 69, 735-9.

de Jonge, P., Ormel, J., van den Brink, R. H., van Melle, J. P., Spijkerman, T. A., Kuijper, A., van Veldhuisen, D. J., van den Berg, M. P., Honig, A., Crijns, H. J. \& Schene, A. H. (2006). Symptom dimensions of depression following myocardial infarction and their relationship with somatic health status and cardiovascular prognosis. Am J Psychiatry 163, 138-44.

de Miranda Azevedo, R., Roest, A. M., Hoen, P. W. \& de Jonge, P. (2014). Cognitive/affective and somatic/affective symptoms of depression in patients with heart disease and their association with cardiovascular prognosis: a meta-analysis. Psychol Med 44, 2689-703.

Denollet, J., Freedland, K. E., Carney, R. M., de Jonge, P. \& Roest, A. M. (2013). Cognitive-affective symptoms of depression after myocardial infarction: different prognostic importance across age groups. Psychosom Med 75, 701-8.

Freedland, K. E. \& Carney, R. M. (2013). Depression as a risk factor for adverse outcomes in coronary heart disease. BMC Med 11, 131.

Haddad, M., Walters, P., Phillips, R., Tsakok, J., Williams, P., Mann, A. \& Tylee, A. (2013). Detecting depression in patients with coronary heart disease: a diagnostic evaluation of the PHQ-9 and HADS-D in primary care, findings from the UPBEAT-UK study. PLoS One 8, e78493.

Hoen, P. W., Conradi, H. J., Denollet, J., Martens, E. J. \& de Jonge, P. (2010a). Interview-based ratings of somatic and cognitive symptoms of depression and their impact on cardiovascular prognosis. Psychother Psychosom 79, 319-20.

Hoen, P. W., Whooley, M. A., Martens, E. J., Na, B., van Melle, J. P. \& de Jonge, P. (2010b). Differential associations between specific depressive symptoms and cardiovascular prognosis in patients with stable coronary heart disease. J Am Coll Cardiol 56, 838-44. 
Kaup, A. R., Byers, A. L., Falvey, C., Simonsick, E. M., Satterfield, S., Ayonayon, H. N., Smagula, S. F., Rubin, S. M. \& Yaffe, K. (2016). Trajectories of Depressive Symptoms in Older Adults and Risk of Dementia. JAMA Psychiatry 73, 525-31.

Kroenke, K., Spitzer, R. L. \& Williams, J. B. (2001). The PHQ-9: validity of a brief depression severity measure. J Gen Intern Med 16, 606-13.

Lamarca, R., Alonso, J., Gomez, G. \& Munoz, A. (1998). Left-truncated data with age as time scale: an alternative for survival analysis in the elderly population. J Gerontol A Biol Sci Med Sci 53, M33743.

Lewis, G., Pelosi, A. J., Araya, R. \& Dunn, G. (1992). Measuring psychiatric disorder in the community: a standardized assessment for use by lay interviewers. Psychol Med 22, 465-86.

Li, H., Van Halm-Lutterodt, N., Zheng, D., Liu, Y., Guo, J., Feng, W., Li, X., Wang, A., Liu, X., Tao, L., Hou, C., Luo, Y., Zhang, F., Yang, X., Gao, Q., Wang, W., Tang, Z. \& Guo, X. (2018). Time-dependent depressive symptoms and risk of cardiovascular and all-cause mortality among the Chinese elderly: The Beijing Longitudinal Study of Aging. J Cardiol 72, 356-362.

Linke, S. E., Rutledge, T., Johnson, B. D., Vaccarino, V., Bittner, V., Cornell, C. E., Eteiba, W., Sheps, D. S., Krantz, D. S., Parashar, S. \& Bairey Merz, C. N. (2009). Depressive symptom dimensions and cardiovascular prognosis among women with suspected myocardial ischemia: A report from the National Heart, Lung, and Blood Institute-sponsored Women's Ischemia Syndrome Evaluation. Arch Gen Psychiatry 66, 499-507.

Martens, E. J., Denollet, J., Pedersen, S. S., Scherders, M., Griez, E., Widdershoven, J., Szabo, B., Bonnier, H. \& Appels, A. (2006). Relative lack of depressive cognitions in post-myocardial infarction depression. J Affect Disord 94, 231-7.

Martens, E. J., Hoen, P. W., Mittelhaeuser, M., de Jonge, P. \& Denollet, J. (2010). Symptom dimensions of post-myocardial infarction depression, disease severity and cardiac prognosis. Psychol Med 40, 807-14.

Meijer, A., Conradi, H. J., Bos, E. H., Thombs, B. D., van Melle, J. P. \& de Jonge, P. (2011). Prognostic association of depression following myocardial infarction with mortality and cardiovascular events: a meta-analysis of 25 years of research. Gen Hosp Psychiatry 33, 203-16.

Meijer, A., Zuidersma, M. \& de Jonge, P. (2013). Depression as a non-causal variable risk marker in coronary heart disease. BMC Med 11, 130.

Moise, N., Khodneva, Y., Jannat-Khah, D. P., Richman, J., Davidson, K. W., Kronish, I. M., Shaffer, J. \& Safford, M. M. (2018). Observational study of the differential impact of time-varying depressive symptoms on all-cause and cause-specific mortality by health status in community-dwelling adults: the REGARDS study. BMJ Open 8, e017385.

National Statistics (30 September 2015). English indices of deprivation 2015 Ministry of Housing, Communities \& Local Government 30 September 2015.

Ormel, J. \& de Jonge, P. (2011). Unipolar depression and the progression of coronary artery disease: toward an integrative model. Psychother Psychosom 80, 264-74. 
Palacios, J., Khondoker, M., Mann, A., Tylee, A. \& Hotopf, M. (2018). Depression and anxiety symptom trajectories in coronary heart disease: Associations with measures of disability and impact on 3-year health care costs. J Psychosom Res 104, 1-8.

Palacios, J. E., Khondoker, M., Achilla, E., Tylee, A. \& Hotopf, M. (2016). A Single, One-Off Measure of Depression and Anxiety Predicts Future Symptoms, Higher Healthcare Costs, and Lower Quality of Life in Coronary Heart Disease Patients: Analysis from a Multi-Wave, Primary Care Cohort Study. PLoS One 11, e0158163.

Pequignot, R., Dufouil, C., Prugger, C., Peres, K., Artero, S., Tzourio, C. \& Empana, J. P. (2016). High Level of Depressive Symptoms at Repeated Study Visits and Risk of Coronary Heart Disease and Stroke over 10 Years in Older Adults: The Three-City Study. J Am Geriatr Soc 64, 118-25.

Rabin, R. \& de Charro, F. (2001). EQ-5D: a measure of health status from the EuroQol Group. Ann Med 33, 337-43.

Roest, A. M., Carney, R. M., Freedland, K. E., Martens, E. J., Denollet, J. \& de Jonge, P. (2013). Changes in cognitive versus somatic symptoms of depression and event-free survival following acute myocardial infarction in the Enhancing Recovery In Coronary Heart Disease (ENRICHD) study. J Affect Disord 149, 335-41.

Roest, A. M., Thombs, B. D., Grace, S. L., Stewart, D. E., Abbey, S. E. \& de Jonge, P. (2011). Somatic/affective symptoms, but not cognitive/affective symptoms, of depression after acute coronary syndrome are associated with 12-month all-cause mortality. J Affect Disord 131, 158-63.

Rose, G. A. (1962). The diagnosis of ischaemic heart pain and intermittent claudication in field surveys. Bull World Health Organ 27, 645-58.

Scafato, E., Galluzzo, L., Ghirini, S., Gandin, C., Rossi, A., Solfrizzi, V., Panza, F., Di Carlo, A., Maggi, S., Farchi, G. \& Group, I. W. (2012). Changes in severity of depressive symptoms and mortality: the Italian Longitudinal Study on Aging. Psychol Med 42, 2619-29.

Smolderen, K. G., Spertus, J. A., Reid, K. J., Buchanan, D. M., Krumholz, H. M., Denollet, J., Vaccarino, V. \& Chan, P. S. (2009). The association of cognitive and somatic depressive symptoms with depression recognition and outcomes after myocardial infarction. Circ Cardiovasc Qual Outcomes 2, 328-37.

Tully, P. J., Winefield, H. R., Baker, R. A., Turnbull, D. A. \& de Jonge, P. (2011). Confirmatory factor analysis of the Beck Depression Inventory-II and the association with cardiac morbidity and mortality after coronary revascularization. J Health Psychol 16, 584-95.

Tylee, A., Ashworth, M., Barley, E., Brown, J., Chambers, J., Farmer, A., Fortune, Z., Haddad, M., Lawton, R., Mann, A., Mehay, A., McCrone, P., Murray, J., Leese, M., Pariante, C. M., Rose, D., Rowlands, G., Smith, A. \& Walters, P. (2011). Up-beat UK: a programme of research into the relationship between coronary heart disease and depression in primary care patients. BMC Fam Pract 12, 38.

Walters, P., Barley, E. A., Mann, A., Phillips, R. \& Tylee, A. (2014). Depression in primary care patients with coronary heart disease: baseline findings from the UPBEAT UK study. PLoS One 9, e98342.

Zigmond, A. S. \& Snaith, R. P. (1983). The hospital anxiety and depression scale. Acta Psychiatr Scand 67, 361-70. 
Table 1. Sample description at baseline $(\mathrm{N}=595)$

\begin{tabular}{|c|c|c|}
\hline Variable & $\mathbf{N}$ & $\%$ \\
\hline Age at cohort entry* & 595 & 72 [27-98] \\
\hline Sex (Male) & 420 & 70.6 \\
\hline Education (> 10 years) & 309 & 51.9 \\
\hline Ethnicity (White) & 530 & 89.1 \\
\hline Living alone (Yes) & 211 & 35.5 \\
\hline \multicolumn{3}{|l|}{ Smoking status } \\
\hline Never & 178 & 29.9 \\
\hline Ex & 330 & 55.5 \\
\hline Current & 87 & 14.6 \\
\hline \multicolumn{3}{|l|}{ Alcohol (units per week) } \\
\hline Doesn't drink & 160 & 26.9 \\
\hline $0-10$ units & 286 & 48.1 \\
\hline$>10$ units & 149 & 25.0 \\
\hline \multicolumn{3}{|l|}{ Body mass index } \\
\hline Normal & 133 & 22.4 \\
\hline Overweight & 272 & 45.7 \\
\hline Obese & 190 & 31.9 \\
\hline \multicolumn{3}{|l|}{ Chronic diseases: } \\
\hline Diabetes & 147 & 24.7 \\
\hline Hypertension & 326 & 54.8 \\
\hline Arthritis & 98 & 16.5 \\
\hline COPD & 65 & 10.9 \\
\hline active Cancer & 67 & 11.3 \\
\hline CKD & 113 & 19.0 \\
\hline Asthma & 42 & 7.1 \\
\hline \multicolumn{3}{|l|}{ Chest pain } \\
\hline No angina & 451 & 75.9 \\
\hline Grade 1 angina & 86 & 14.5 \\
\hline Grade 2 angina & 58 & 9.6 \\
\hline \multicolumn{3}{|l|}{ Social problems } \\
\hline No problems & 281 & 47.2 \\
\hline 1 problem & 197 & 33.1 \\
\hline 2 to 7 problems & 117 & 19.7 \\
\hline \multicolumn{3}{|l|}{ Diagnosis at registry entry } \\
\hline Myocardial infarction & 252 & 42.4 \\
\hline Ischemic heart disease & 287 & 48.2 \\
\hline Other & 56 & 9.4 \\
\hline Depression diagnosis (CIS-R) & 595 & 5.9 \\
\hline PHQ-9 score $(>10)$ & 595 & 16.8 \\
\hline PHQ-9 somatic dimension* & 595 & $2[0-12]$ \\
\hline PHQ-9 cognitive dimension*\# & 595 & $0[0-12]$ \\
\hline Age registry entry* & 595 & 60.6 [25.6-97.2] \\
\hline Quality of life score* & 595 & $3[3-8]$ \\
\hline Anxiety score* & 595 & $4[0-21]$ \\
\hline IMD* ${ }^{*}$ & 593 & $16.9[1.8-62.4]$ \\
\hline
\end{tabular}

COPD: chronic obstructive pulmonary disease; CKD: chronic kidney disease; IMD: Index of Multiple Deprivation *median [min-max]

\#standardised to a scale of 0-12 for comparative purposes 
Table 2. Associations between baseline and time-dependent depressive symptom dimensions and new cardiac event at follow-up (95 events/595)

\begin{tabular}{cclcc}
\hline & Baseline analysis & & $\begin{array}{l}\text { Time-dependent } \\
\text { analysis }\end{array}$ & \\
\hline & $\mathrm{HR}(95 \% \mathrm{Cl})$ & $\mathrm{p}$ & $\mathrm{HR}(95 \% \mathrm{Cl})$ & $\mathrm{p}$ \\
\hline $\begin{array}{c}\text { Univariate model } \\
\quad \text { Somatic dimension }\end{array}$ & $1.13(1.05-1.21)$ & 0.0005 & $1.12(1.04-1.19)$ & 0.001 \\
$\quad$ Cognitive dimension & $1.09(1.02-1.16)$ & 0.01 & $1.09(1.02-1.16)$ & 0.01 \\
& & & & \\
Multivariate model $^{2}$ & & & & 0.001 \\
$\quad$ Somatic dimension & $1.13(1.06-1.22)$ & 0.0007 & $1.12(1.05-1.20)$ & 0.004 \\
$\quad$ Cognitive dimension & $1.09(1.02-1.17)$ & 0.01 & $1.11(1.03-1.18)$ & \\
\hline
\end{tabular}

\footnotetext{
1 adjusted for sex

2 further adjusted for diagnosis at entry (MI no/yes), smoking, and time-dependent chest pain, hypertension, COPD, active cancer
} 
Table 3. Association between baseline and time-dependent PHQ-9 items and NCE (multivariate Cox Model ${ }^{1}$ )

\begin{tabular}{lclcl}
\hline $\mathrm{n} / \mathrm{N}=95 / 595$ & $\begin{array}{l}\text { Baseline } \\
\text { depression }\end{array}$ & \multicolumn{3}{c}{$\begin{array}{l}\text { Time-dependent } \\
\text { Depression }\end{array}$} \\
\hline & HR (95\% Cl) & $\mathrm{p}$ & $\mathrm{HR}(95 \% \mathrm{Cl})$ & $\mathrm{p}$ \\
\hline Somatic dimension items (item, $\left.\mathbf{n}^{\circ}\right):$ & & & & \\
$\begin{array}{l}\text { Trouble with sleep (3) } \\
\text { Feeling tired or having little energy (4) }\end{array}$ & $1.18(0.98-1.41)$ & 0.08 & $1.23(1.03-1.46)$ & 0.03 \\
Poor appetite or overeating (5) & $1.26(1.03-1.53)$ & 0.02 & $1.20(0.99-1.44)$ & 0.06 \\
Slowness or restlessness (8) & $1.40(1.15-1.71)$ & 0.0008 & $1.31(1.07-1.60)$ & 0.009 \\
& $1.38(1.07-1.77)$ & 0.01 & $1.30(1.03-1.65)$ & 0.03 \\
Cognitive dimension items (item, $\left.\mathbf{n}^{\circ}\right):$ & & & & \\
Little interest/pleasure in doing things (1) & $1.31(1.05-1.63)$ & 0.02 & $1.26(1.01-1.57)$ & 0.04 \\
Feeling down, depressed or hopeless (2) & $1.37(1.07-1.74)$ & 0.01 & $1.32(1.05-1.66)$ & 0.02 \\
Feeling like a failure (6) & $1.27(0.98-1.65)$ & 0.07 & $1.39(1.08-1.79)$ & 0.01 \\
Trouble concentrating (7) & $1.15(0.90-1.49)$ & 0.3 & $1.25(0.99-1.58)$ & 0.06 \\
Thoughts that better off dead (9) & $1.25(0.88-1.75)$ & 0.2 & $1.29(0.87-1.91)$ & 0.2 \\
\hline
\end{tabular}

\footnotetext{
${ }^{1}$ adjusted for sex, diagnosis at entry (MI no/yes), smoking, and time-dependent chest pain, hypertension, COPD, active cancer
} 